\title{
Performance Evaluation of Network Gateway Design for NoC based System on FPGA Platform
}

\author{
Guruprasad S.P ${ }^{1}$ \\ Research Scholar, Dept. of ECE \\ Jain University, Bangalore, India
}

\author{
Dr.Chandrasekar B.S ${ }^{2}$ \\ Director, CDEVL \\ Jain University, Bangalore, India
}

\begin{abstract}
Network on Chip (NoC) is an emerging interconnect solution with reliable and scalable features over the System on Chip (SoC) and helps to overcome the drawbacks of bus-based interconnection in SoC. The multiple cores or other networks have a boundary which is limited to communicate with devices, which are directly connected to it. To communicate with these multiple cores outside the boundary, the NOC requires the gateway functionality. In this manuscript, a cost-effective Network Gateway (NG) model is designed, and also the interconnection of a network gateway with multiple cores are connected to the NoC based system is prototyped on Artix-7 FPGA. The NG mainly consists of Serializer and deserializer for transmitting and receiving the data packets with proper synchronization, temporary register to hold the network data, electronic crossbar switch is connected with multiple cores which are controlled by switch controller. The NG with the Router and different sizes of NoC based system is designed using congestionfree adaptive-XY routing. The implementation results and performance evaluation are analyzed for NG based NoC in terms of average Latency and maximum Throughput for different Packet Injection Ratio (PIR). The proposed Network gateway achieves low latency and high throughput in NoC based systems for different PIR.
\end{abstract}

Keywords-Network gateway; network on chip; FPGA; routing; network interface; crossbar switch

\section{INTRODUCTION}

The NoC will play an emerging role in future highperformance Chip Multi-Processor (CMP) to address the problems of interconnections. In recent years, most of the research focused on a packet-switched NoC design, which improves the system performance by using optimization techniques in the network for better Latency and bandwidth and also supports on-chip and off-chip communications. The NoC based photonic communications support a mechanism for large data transmission with higher bandwidth and less power consumption. The photonic based NoC supports Multiple cores interface using gateway switch [1] [3]. Multiple cores residing in a single chip (MPSoC) exist towards mixed-criticality system includes dependability, security, and different block access with shared resources. The outside network real-time messages are communicating to MPSoC using a gateway [2]. In general, the network gateway is a node which connects two different networks with different transmission protocols and simplifies the internet connectivity into one electronic device. The gateway node acts as a firewall and proxy server for business use. Gateways are a protocol which provides the compatibility between two different protocols and will be operating in any of the Open system interconnection (OSI) layers. The multifunctional intercommunication supported by the Gateway on a single-core chip. The different protocol standards like Bluetooth, Modbus, serial bus, Process Filed bus, and Controller area Network (CAN) provides intercommunication using gateway [4]. The intelligent Gateway has interoperation and achieves better communication among different bus networks with reconfigurability and also supports fast conversion speed, flexibility, intellectual control ability, reliability, and higher-level interface. The protocol converting Gateway works on Most of the OSI layers [5-6]. The high-performance computation needs high-speed interconnection like Ethernet and Infiniband. The data transmission between two heterogeneous networks needs an efficient network gateway to improve system performance in terms of bandwidth and Latency [7] [12].

The gateway terminology is used commonly for most of the applications for protocol conversion and data packets transfers. The network gateways are used in most of the real-time embedded and Internet of Things (IoT) applications. The home gateway requires a standard ARM chip with SoC chip which integrates the Customer Electronics Bus (CEbus) with home appliances like TV, microwave oven, refrigerator, and washing machine. The user sends a command to the internet; the network control module receives the command, issues request signal to Chip to control the home appliances [8]. The heterogeneous Gateway provides different interfaces to internet, GSM, CDMS, PSTN, and so on, to support different application scenarios [9]. The embedded Gateway is a backbone for smart grid home networks [10], wireless applications [11] [14], indoor high precision positioning systems [13], and IoT applications [15] for communicating with other networks.

In this manuscript, a cost-effective Network Gateway model is designed along with Gateway based NoC system using Adaptive XY Routing. The Network gateway results are hardware resource-efficient, works at low Latency, and High Throughput for input traffics which are evaluated for NoC based system. Section II explains about related work on Gateway mechanism used for different applications and also explains about research findings. Section III elaborates the Network Gateway architecture using electronic crossbar switch with an explanation. Section IV explains the Network Gateway based NoC based system with router architecture. The results and performance evaluation are analyzed with tables and graphs in Section V. Finally concludes the overall proposed work with Future scope in Section VI. 


\section{RELATED WORKS}

In this section, the general Gateway related work and applications of Gateway are reviewed. Shi et al. [16] presented an embedded dual home architecture with secured Gateway both on hardware and software platform. The Gateway improves the transmitting information risk by the user and network isolation module using FPGA is incorporated to improve the security features using data signature and key management. The secured embedded Virtual private network (VPN) gateway is presented by Han et al. [17] to improve the data transmission security with protection capability in application terminals. This VPN gateway is worked under L3, L4, and L7 layers with firewall protection, VPN Functioning, and network isolation modules. Ajami et al. [18] presented an FPGA Based embedded network firewall which supports highly customized data packet filtering on a network gateway. These firewall customized in real-time by changing the TCP/UDP port id, Source MAC address, and sourcedestination IP address. Abuteir et al. [19] introduced a gateway design to establish the hierarchical platform for multi-core chips interaction either on on-chip or off-chip networks. The software-based Gateway supports message classification, message - scheduling, traffic shaping services, downsampling, service, protocol conversion, egress-queuing, ingress-Queuing, Virtual-Link queuing, and also supports serialization services.

Obermaisser et al. [20] described the mixed-criticality systems for end to end real-time communication, which involves gateways between multiple off-chip networks, Gateway between off-chip and on-chip networks. The gateway node resolving the contention between source controlled and autonomous networks and also supports end-to-end addressing and routing. The cloud storage gateway was presented by Dumitru et al. [21] on FPGA platform. The secured data encryption and transparency are resolved by using FPGA between host and outside interface in cloud infrastructure. Lee et al. [22] presented a high-performance hardware-software based gateway design for In-Vehicle Network (IVN) for CAN/ FlexRay controllers. The data conversion between CAN to FlexRay and vice-versa is achieved using Routing table converter block with AXI interface on Zed board. Shreejith et al. [23] described the vehicular Ethernet Gateway connected with multiple network protocols like FlexRay, CAN, and Ethernet with embedded computing Units. The Ethernet gateway is designed using Switch fabric between FlexRay and Ethernet controller. The switch fabric is designed using Crossbar switch.

The embedded Gateway for Fourth Generation (4G) mobile network and process Fieldbus (PB) with decentralized Periphery (DP) is described by Zhou et al. [24] on FPGA platform. The AES algorithm is used for secured data transaction in Gateway. The Gateway is used to connect two different protocol $4 \mathrm{G}$ and $\mathrm{PB}$ conversion in terms of data. The Korona et al. [25] introduced an Internet Protocol security (IPsec) gateway for multi-gigabit networks which includes security association database to store secure information, Internet key exchange to set secure channels, and responsible for all security operation with packet encapsulation. The programmable-SoC (PSoC) based cyber-physical production system (CPPS) gateway is described by Urbina et al. [26] to meet the industry 4.0 standards. The industrial network architecture includes CPPS Gateways, which are interconnected with multiple peripherals, electronic and electrical devices using different network protocols like Profinet, Profibus, and High availability Seamless Redundancy (HSR). Kwak et al. [27] present the trust domain gateway system to solve the untrusted internet structural problems.

Gaps in the research: Most of the work carried on traditional software-based gateway designs lacks with latency and throughput issues. Hardware-based Network gateway designs use bus-based interconnections for embedded real-time applications and lack of scalability and reliability problems. The existing research work is done on protocol conversion using gateways, but not on NoC based system. In order to resolve these problems, a cost-effective Network gateway with NoC based system is designed.

\section{NETWORK GATEWAY DESIGN}

The Gateway provides the network and access information to the four gateway cores, and the hardware architecture of the network gateway is represented in Fig. 1. The network gateway mainly consists of deserializer and Serializer for receiving and transmitting the data information's with proper synchronization, Temporary register, Electronic crossbar switch, Switch controller, priority encoder, and four gateway cores. The gateway cores are processors, buffers, caches, peripheral devices, etc. The FIFO buffers are considered in the design.

The data information is received from the network either from the interface or from the Router to deserializer, which receives the data signals serially, works based on Serial In Parallel Out (SIPO) manner. The received 8-bit data converts to 32-bit data to parallel using shifting operation along with issuing the synchronization signal to Serializer. The synchronization is achieved between Serializer and deserializer using counter method and proper clocking mechanism. The temporary register receives the deserialized data, holds for access to the electronic crossbar. This temporary register is only used to store the received deserialized data signals and that are scheduled towards for the gateway cores through switch controller. The electronic crossbar switch receives the temporary data along with gateway core (buffer) inputs and works based on switch controller, and its hardware architecture is represented in Fig. 2.

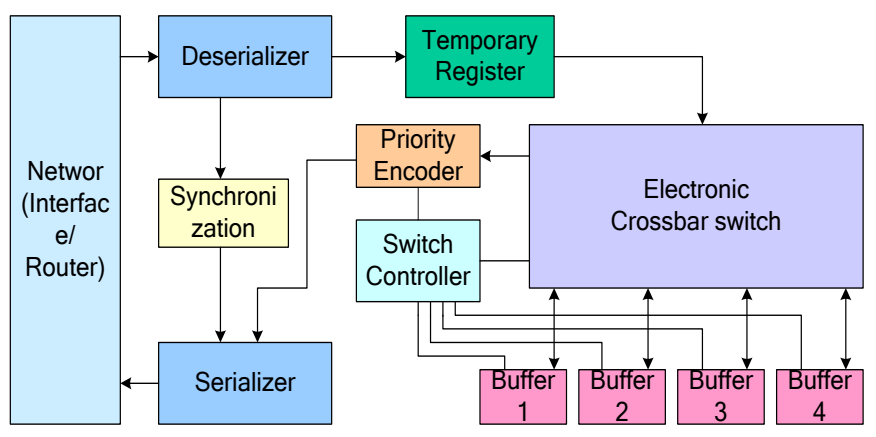

Fig. 1. Hardware Architecture of Network Gateway. 


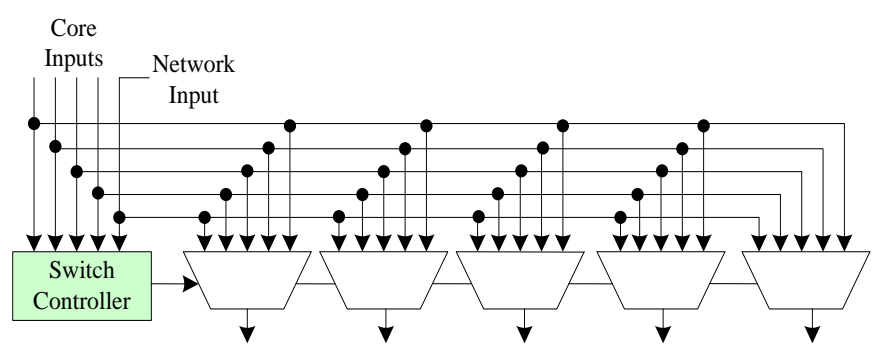

Fig. 2. Electronic Crossbar Switch Diagram.

The electronic crossbar switch have mainly five multiplexors, and each multiplexer is five inputs, four from the gateway cores and one from the temporary register (which is coming from the network). The switch controller issues the select line based time slot and priority to the crossbar switch and generates the prioritize output. The switch controller receives the five request inputs from the gateway cores and temporary register. The switch controller works based on the arbitration and time slot. The controlling mechanism is incorporated in the switch controller using Finite State Machine (FSM), which receives the input requests, gives priority to the corresponding input and other requests in waiting for the state.

The electronic crossbar issues the data signals based switch controller to priority encoder as an input. The same select signal issue the prior encoded data signal to Serializer. The Serializer is ready to transmit the data signals to the network form the crossbar switch. The Serializer converts the 32-bit parallel data information to 8-bit serially in PISO manner with synchronization and sends to network. The received and transmitted data of the network is same in the Gateway, which proves that the designed Gateway is working effectively.

\section{NETWORK GATEWAY FOR NOC SySTEM}

The network gateway is interconnected to the NoC based systems which offer on-chip and off-chip data flow control and arbitration between many gateway cores interconnections of the NoC based Multiprocessing SoC (MPSoC). The MPSoC chips are considered as an FPGA or ASIC devices for prototyping the network gateway with NoC. The network Gateway interconnected to NoC, and it is represented in Fig. 3. This is an example of $4 \times 4$ Mesh topology-based Network gateways with NoC Connection. It mainly contains 16 routers, 16 network gateways with 64-processing cores and all are interconnected with linked wires. This architecture is flexible to support any of the64- processing core information's that can transmit to any of the 16 routers via network gateways using Adaptive routing algorithm.

The network gateway with cores is connected to routers via a network interface (NI). In design, Mesh topology is selected to design $2 \times 2,3 \times 3$, and $4 \times 4$ NoC architectures. In Fig. 3, the $4 \times 4$ NoC has 16 routers ( $R 1$ to $R 16$ ), and all the routers are interconnected using linked wires. All the network Gateway with cores inputs are received to the corresponding routers via the network interface and perform the data transaction based on the destination address of the corresponding routers.

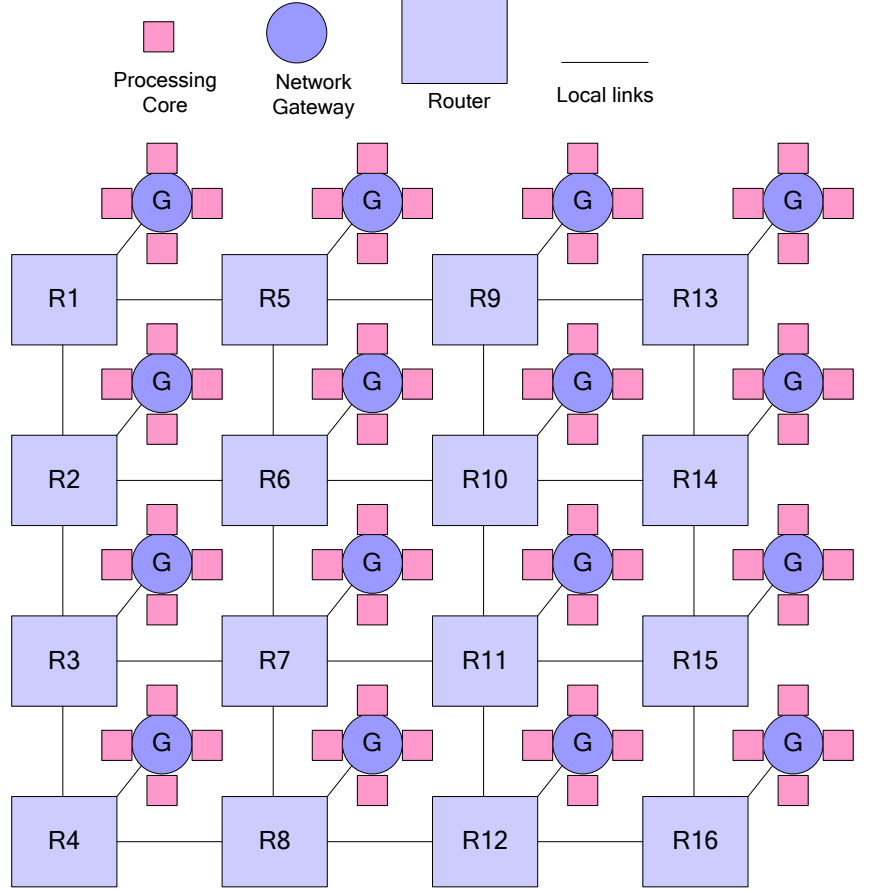

Fig. 3. Network Gateway based NoC Design Architecture.

The router architecture of Network Gateway is represented in Fig. 4. The designed Router is congestion-free Router which finds the shortest route to reach the destination. Each Router has five-port input registers followed by packet formation with priority-based arbitration and adaptive XY routing algorithm. The five-port input register receives gateway data information and stores it in local input port (Li), and For NoC, supported service inputs are East (Ei), West (Wi), South (Si) and North $(\mathrm{Ni})$ are presented to route to corresponding destination locations.

The 8-bit local gateway data are used for packet formation along with user address and request input. The Network Gateway based Router packet formation is represented in Fig. 5. The packet is framed based on a request, destination address provided by the user, and gateway input. So The NoC is having a 13-bit packet which includes 1-bit request, 2-bit destination $\mathrm{X}$ address, 2-bit destination $\mathrm{Y}$ address, and 8-bit Gateway data.

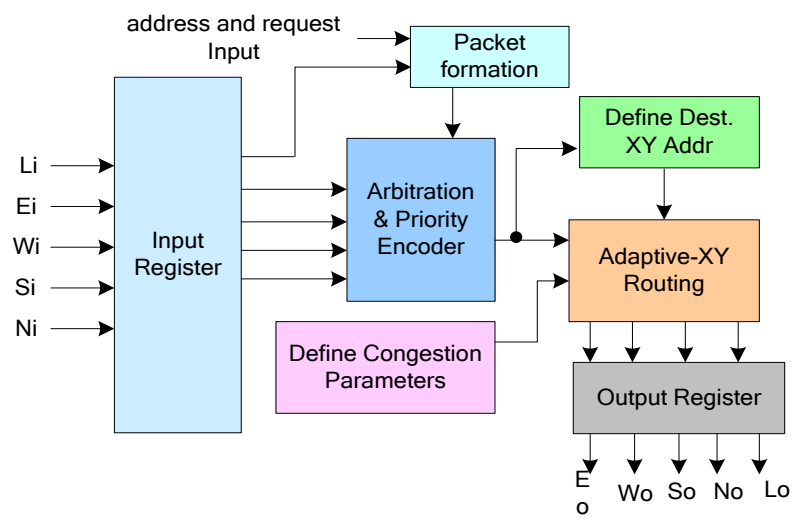

Fig. 4. Hardware Architecture of Network Gateway based Router. 


\begin{tabular}{|c|l|l|c|}
\hline 1-bit & 2-bits & 2-bits & 8-bits \\
\hline Request & Dest.X & Dest.Y & Gateway Data \\
\hline
\end{tabular}

Fig. 5. Packet formation for Network Gateway based Router.

The framed packet, along with four more from input register is input to priority encoder. The priority encoder works based on the arbitration. The Arbiter receives the MSB bits from all the five ports and considered as requests and generates the 5-bit grants based on the priority. These grants are acts as a select line to priority encoder. The encoded data is a prioritize packet data, and it sends as an input to the adaptive routing-XY algorithm. Each Router, R1 to R16, has fixed 4-bit current XY address and which is easy to identify the Router. For example, in design, R4 is set to "0011," and R14 is " 1101 ".

To perform the routing computation, first, define the congestion parameters along with Destination-XY address from the encoded packet. The adaptive- $X Y$ routing is congestion-free routing and adaptive form of normal $-\mathrm{XY}$ routing [28]. The $\mathrm{X}$ or $\mathrm{Y}$ direction with less number of routing path is defined and the routing packet id assisted to the destination with less congestion. Based on congestion parameters, which finds the shortest routing path to reach the destination with less traffic. The Network Gateway based single router, $2 \mathrm{X} 2,3 \mathrm{X} 3$, and $4 \mathrm{X} 4 \mathrm{NoC}$ 's are designed and prototyped on FPGA, which are explained detail in the next section.

\section{RESUlts AND PERFORMANCE ANALYSIS}

The results and performance evaluation are analyzed in this section for Network Gateway (NG) Module, and NG Based NoC using Mesh topology. The NG and NG-Based NoC are designed using Verilog-HDL on Xilinx platform and implemented on Artix-7 FPGA.

\section{A. Implementation Results}

The Network gateway implementation results after a place and route process on Artix-7 FPGA are tabulated in Table I. The resources in terms of Area-Slices, LUT's, Design operating frequency, and total power utilized are represented. The NG utilizes 450 slice registers, 893 slice LUTs and operating at 319.642 MHz frequency. The NG utilizes $0.104 \mathrm{~W}$ total power, which includes $0.022 \mathrm{~W}$ dynamic power using X-power analyzer.

The NG Module is designed for NoC based Multiprocessing SoC applications. The NG Based Router is designed using Adaptive $-X Y$ routing algorithm. The different network sizes like $2 \mathrm{X} 2,3 \mathrm{X} 3$, and, $4 \mathrm{X} 4$ are designed using mesh topology. The Chip area utilization for NG Based NoC designs are represented in Table II. The graphical visualization of the NG based NoC designs for area utilization is represented in Fig. 6.

The total power (W) analysis of NG based NoC design with respect to Different clock frequencies are represented in Fig. 7.

The Power analysis results are generated using Xilinx XPower analyzer and the ambient temperature, and the initial source voltage is set to $25^{\circ} \mathrm{C}$ and 1 Volt, respectively. The NG router and NG-4X4 NoC utilizes $1.032 \mathrm{~W}$ and $1.10 \mathrm{~W}$ respectively for $5000 \mathrm{MHz}$ clock frequency. The network gateway based NoC designs are implemented effectively on FPGA with better chip area, speed, and power tradeoffs have been achieved.

TABLE. I. NETWORK GATEWAY RESOURCE IMPLEMENTATION RESUlTS

\begin{tabular}{|l|l|}
\hline Resources & Utilized on Artix-7 FPGA \\
\hline Slice Registers & 450 \\
\hline Slice LUTs & 893 \\
\hline LUT-Flipflops & 252 \\
\hline Max. Frequency (MHz.) & 319.642 \\
\hline Total power (W) & 0.104 \\
\hline
\end{tabular}

TABLE. II. RESOURCE UTILIZATION-FOR NG-NOC DESIGN

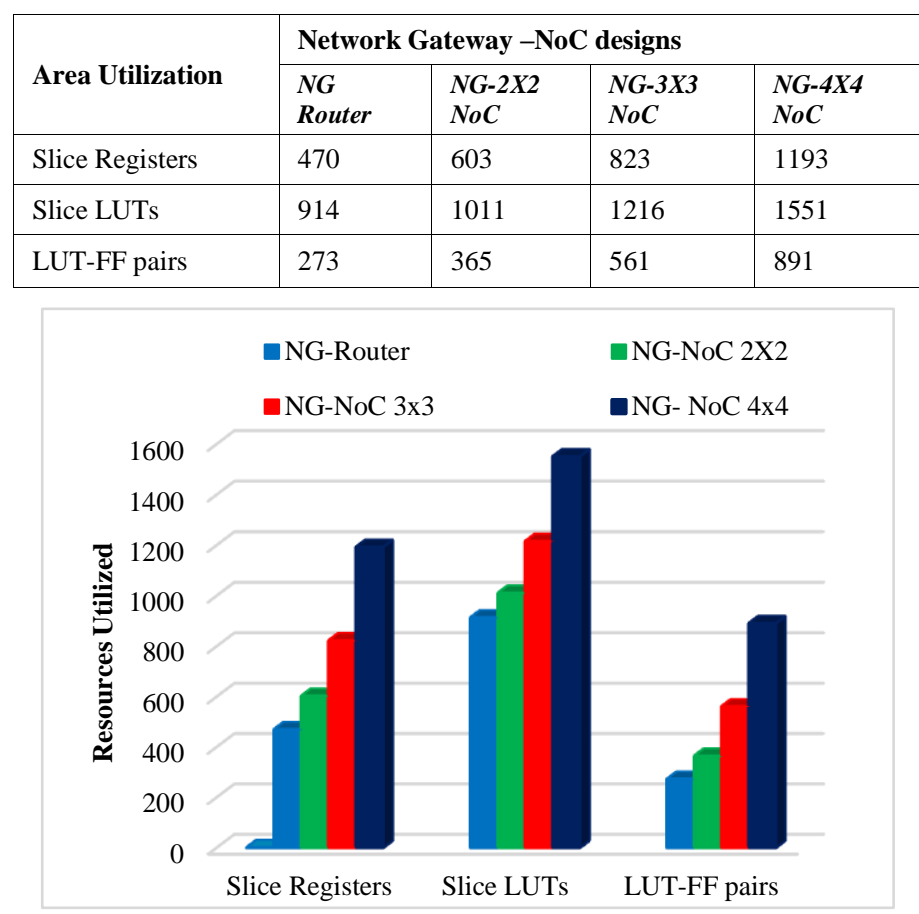

Fig. 6. Network Gateway-NoC Designs Area utilization on Artix-7 FPGA.

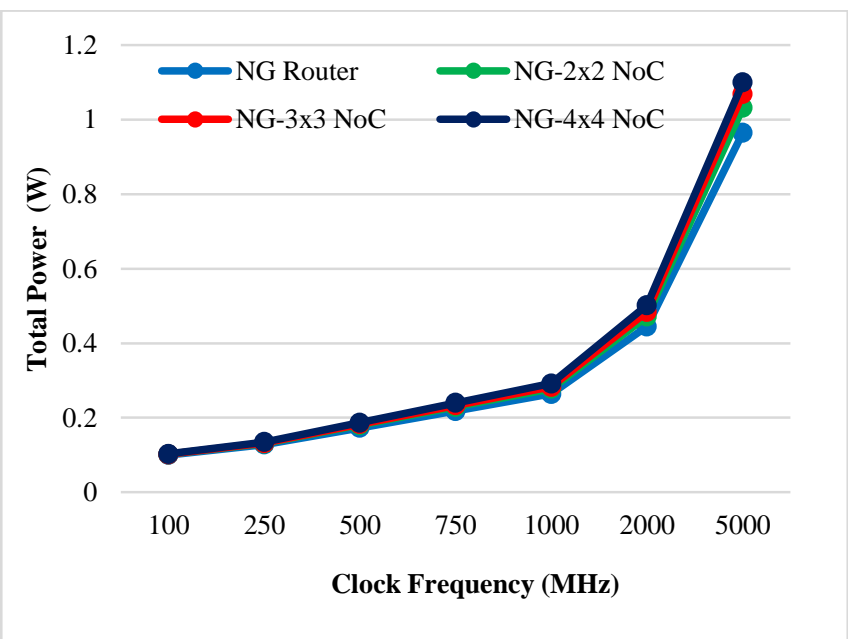

Fig. 7. Total Power v/s different Frequencies for NG based NoC Design. 


\section{B. Performance Evaluation}

The performance analysis of this work is evaluated using average Latency and maximum Throughput with respect to input traffic. The wormhole switching method and uniform traffic patterns are considered for analysis purpose. The Packet Injection Rate (PIR) is defined as the total number of data packets that can be sent on a single clock cycle. The average latency for network gateway is calculated using below equation (1).

$(\text { Avg. Latency })_{\mathrm{NG}}=$ Min.NG latency + No. of Flits

The minimum Network gateway (NG) latency in terms of clock cycles is 18.5 . The number of flits used in the design is 8 . So average latency for NG is 26.5 clock cycles. For the NG Based NoC design, the Average Latency for NG based NoC design is expressed in the below equation (2).

$(\text { Avg. Latency })_{\mathrm{NoC}}=$

$\left(\right.$ No. of PE's * $\left.(\text { Avg. Latency })_{\mathrm{NG}}\right)+($ No. of PE's * 2)

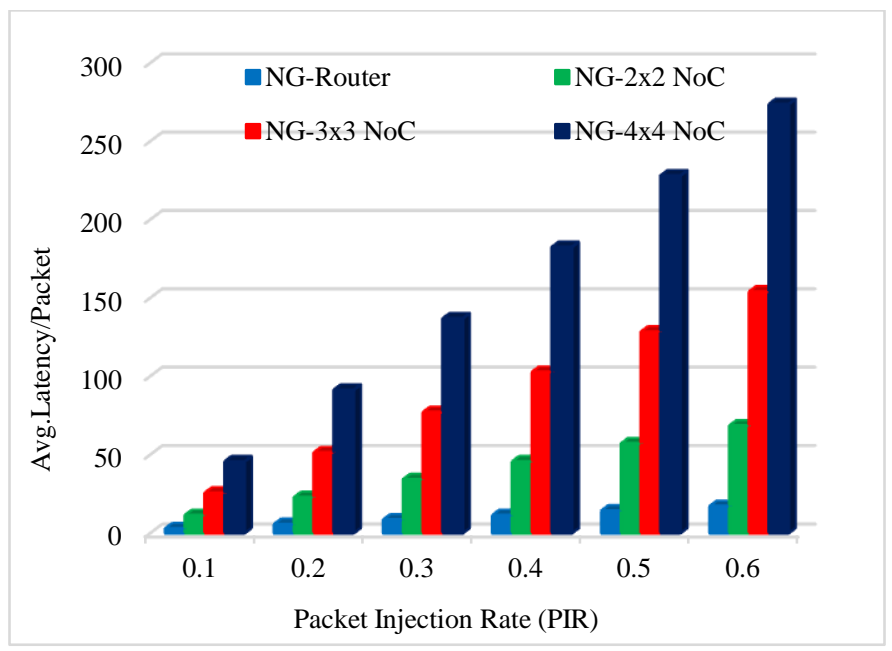

Fig. 8. Average Latency v/s Input Traffic for Network Gateway-NoC Designs using Mesh Topology.

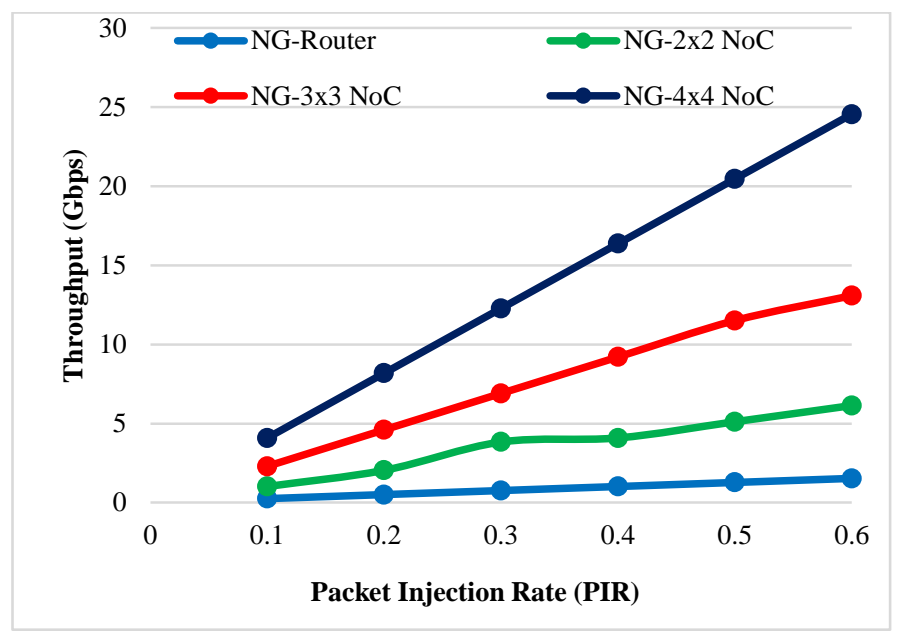

Fig. 9. Max.Throughput v/s Input Traffic for Network Gateway-NoC Designs.
The number of Processing elements (PE's) is defined based on Mesh Topology used in NoC. The 2 clock cycles are considered additionally, which is time taken to forward the packet from source to destination in NoC Network. The input traffic interms of PIR are evaluated in each NG-NoC designs are represented in Fig. 8 for average latency calculation. The average Latency for NG based NoC is represented in terms of Clock cycles (ns).

The maximum Throughput for NG based NoC is defined based on a number of PE's followed by Gateway data width, PIR, and Maximum operating frequency $(\mathrm{MHz})$. And it is represented by using the below equation (3).

$(\text { Throughput })_{\text {NoC }}=$ No.of PE's * Datawidth * PIR * $\mathrm{F}_{\max }$

The PE's are connected NoC boundary via a network interface. The throughput calculation depends upon the data width used in the Gateway. For example, the $4 \mathrm{X} 4 \mathrm{NoC}$ with 8bit data packet are connected 16 PE's and operated on maximum frequency $\left(\mathrm{F}_{\max }\right)$ of gateway design used in artix-7 FPGA. The maximum Throughput with respect to Input traffic is represented in Fig. 9. The maximum Throughput of NGRouter and NG-4X4 NoC operated at 1.5342 Gbps and 24.548 Gbps respectively. The maximum Throughput varies based on data width selection. In design 8-bit data width is selected.

\section{CONCLUSION AND FUTURE WORK}

This manuscript presents an efficient and cost-effective Network Gateway (NG) model using Electronic crossbar switch along with Network gateway in NoC based system. The NG design is flexible to support multiple cores and easy to prototype on on-chip devices. The NG with a single Router and different sizes of NoC using mesh topology is designed using Adaptive $\mathrm{XY}$ routing. The NG implementation results on Artix-7 FPGA utilizes $<1 \%$ hardware resources and NG based $4 X 4$ NoC utilizes $>2 \%$ resources. The NG operates at 319.6 $\mathrm{MHz}$ and consumes less total power around $0.104 \mathrm{~W}$ on FPGA. The Performance analysis of NG based NoC is evaluated using Average Latency and Maximum Throughput with respect to different Input traffic. The average Latency for NG and NG Based 4X4 NoC design utilizes 15.9 and 273.6 at 0.6 PIR, respectively. The maximum Throughput for NG and NG Based 4X4 NoC design works at $1.53 \mathrm{Gbps}$ and $24.54 \mathrm{Gbps}$ at 0.6 PIR respectively for 8-bit data width. This architecture can be incorporated in futuristic researches with the security features to Network Gateway and NoC based systems to strengthen the data packets from attacks.

\section{REFERENCES}

[1] Petracca, Michele, Keren Bergman, and Luca P. Carloni. "Photonic networks-on-chip: Opportunities and challenges." In 2008 IEEE International Symposium on Circuits and Systems, pp. 2789-2792. IEEE, 2008.

[2] Petrakis, Polydoros, Mohammed Abuteir, Miltos D. Grammatikakis, Kyprianos Papadimitriou, Roman Obermaisser, Zaher Owda, Antonis Papagrigoriou, Michael Soulie, and Marcello Coppola. "On-chip networks for mixed-criticality systems." In 2016 IEEE 27th International Conference on Application-specific Systems, Architectures and Processors (ASAP), pp. 164-169. IEEE, 2016.

[3] Hendry, Gilbert, Johnnie Chan, Shoaib Kamil, Lenny Oliker, John Shalf, Luca P. Carloni, and Keren Bergman. "Silicon nanophotonic networkon-chip using TDM arbitration." In 2010 18th IEEE Symposium on High-Performance Interconnects, pp. 88-95. IEEE, 2010. 
[4] Hu, Yonghong, and Lu Ding. "Design and Realization of Multifunctional Gateway Based on Single Chip." In 2009 2nd International Congress on Image and Signal Processing, pp. 1-4. IEEE, 2009.

[5] Guo, Tiantian, Ming' a Zhou, and Qing Shen. "A Reconfigurable Intelligent Gateway for Heterogeneous Networks." In Proceedings of 2013 Chinese Intelligent Automation Conference, pp. 485-493. Springer, Berlin, Heidelberg, 2013.

[6] Guo, Xiaodong, and Haijun Ren. "Multimode communication gateway design in heterogeneous network environments, intelligent distribution, and utilization." In 2015 6th IEEE International Conference on Software Engineering and Service Science (ICSESS), pp. 326-329. IEEE, 2015.

[7] Shi, Wei, Gaofeng Lv, Zhigang Sun, and Zhenghu Gong. "HiTrans: An FPGA-Based Gateway Design and Implementation in HPC Environments." In International Conference on Algorithms and Architectures for Parallel Processing, pp. 561-571. Springer, Cham, 2015.

[8] Ni, Binbin, Mingguang Wu, and Yanpeng Liu. "Design of Embedded Home Network Gateway for CE Bus Based on ARM." In 2006 4th IEEE International Conference on Industrial Informatics, pp. 1380-1384. IEEE, 2006

[9] Luo, Hong, Cheng Chang, and Yan Sun. "Advanced sensor gateway based on FPGA for wireless multimedia sensor networks." In 2011 International Conference on Electric Information and Control Engineering, pp. 1141-1146. IEEE, 2011.

[10] Nguyen, Minh-Triet, Lap-Luat Nguyen, and Tuan-Duc Nguyen. "On the design of gateway node for smart gird home network." In 2015 International Conference on Communications, Management and Telecommunications (ComManTel), pp. 57-61. IEEE, 2015.

[11] Shaofeng, Lin, Tao Bo, Pan Jin, Wan Juan, and Du Jia. "Design and Implementation of Embedded Wireless Gateway." In 2015 International Conference on Intelligent Transportation, Big Data and Smart City, pp. 270-273. IEEE, 2015.

[12] Zheng, Qi. "The design and the implementation of communication gateway between CAN bus and Ethernet." In 2015 IEEE Advanced Information Technology, Electronic and Automation Control Conference (IAEAC), pp. 862-866. IEEE, 2015.

[13] Wang, Wenhua, Weiwei Xia, Rui Zhang, and Lianfeng Shen. "Design and implementation of gateway and server in an indoor high-precision positioning system." In 2014 IEEE 3rd Global Conference on Consumer Electronics (GCCE), pp. 540-541. IEEE, 2014.

[14] Baoxia, Sun, Wang Weixing, Tie Fenglian, and Weng Jiangpeng. "Design and implementation of gateway for hybrid antenna clustering routing algorithm in paddy monitoring." In 2016 6th International Conference on Electronics Information and Emergency Communication (ICEIEC), pp. 310-313. IEEE, 2016.

[15] Zhong, Chang-Le, Zhen Zhu, and Ren-Gen Huang. "Study on the IOT architecture and gateway technology." In 2015 14th International Symposium on Distributed Computing and Applications for Business Engineering and Science (DCABES), pp. 196-199. IEEE, 2015.

[16] Shi, Yonghong, Jianzhong Shen, Lin Zhang, Qian Zhang, and Shaofeng Lin. "Design of Security Gateway Based On Dual-Homed Architecture."
In 2016 International Conference on Robots \& Intelligent System (ICRIS), pp. 159-163. IEEE, 2016.

[17] Kun Han, Junjie Liu, Demin Yang and Quan Yuan, "The design of secure embedded VPN gateway," 2014 IEEE Workshop on Advanced Research and Technology in Industry Applications (WARTIA), Ottawa, ON, 2014, pp. 350-353.

[18] Ajami, Raouf, and Anh Dinh. "Embedded network firewall on FPGA." In 2011 Eighth International Conference on Information Technology: New Generations, pp. 1041-1043. IEEE, 2011.

[19] Abuteir, Mohammed, Romn Obermaisser, Zaher Owda, and Thierry Moudouthe. "Off-chip/on-chip gateway architecture for mixed-criticality systems based on networked multi-core chips." In 2015 IEEE 18th International Conference on Computational Science and Engineering, pp. 120-128. IEEE, 2015.

[20] Obermaisser, Roman, Zaher Owda, Mohammed Abuteir, Hamidreza Ahmadian, and Donatus Weber. "End-to-end real-time communication in mixed-criticality systems based on networked multi-core chips." In 2014 17th Euromicro Conference on Digital System Design, pp. 293302. IEEE, 2014.

[21] Dumitru, Laurențiu A., Sergiu Eftimie, and Dan Fostea. "An FPGABased cloud storage gateway." In 2nd International Conference SEACONF, Academia Navală Mircea Cel Bătrân, Constanța. 2016.

[22] Lee, Trong-Yen, Chia-Wei Kuo, and I-An Lin. "High performance CAN/FlexRay gateway design for in-vehicle network." In 2017 IEEE Conference on Dependable and Secure Computing, pp. 240-242. IEEE, 2017.

[23] Shreejith, Shanker, Philipp Mundhenk, Andreas Ettner, Suhaib A. Fahmy, Sebastian Steinhorst, Martin Lukasiewycz, and Samarjit Chakraborty. "VEGa: A high performance vehicular Ethernet gateway on hybrid FPGA." IEEE Transactions on Computers 66, no. 10 (2017): 1790-1803.

[24] Zhou, Yuan, Wenping Xiao, Mingshan Liu, and Xiaokun Li. "Design of the embedded gateway for $4 \mathrm{G}$ and PROFIBUS-DP based on FPGA." In 2017 3rd IEEE International Conference on Computer and Communications (ICCC), pp. 748-752. IEEE, 2017.

[25] Korona, Mateusz, Krzysztof Skowron, Mateusz Trzepiński, and Mariusz Rawski. "FPGA implementation of IPsec protocol suite for multigigabit networks." In 2017 International Conference on Systems, Signals and Image Processing (IWSSIP), pp. 1-5. IEEE, 2017.

[26] Urbina, Marcelo, Armando Astarloa, Jesús Lázaro, Unai Bidarte, Igor Villalta, and Mikel Rodriguez. "Cyber-physical production system gateway based on a programmable SoC platform." IEEE Access 5: 20408-20417, 2017.

[27] Kwak, Byeong Ok, and Tae Soo Chung. "Design and Implementation of the Trust Domain Gateway System." In 2018 International Conference on Information and Communication Technology Convergence (ICTC), pp. 925-927. IEEE, 2018.

[28] Guruprasad, S. P., and B. S. Chandrasekar, "Design and Performance analysis of Adaptive-XY over $\mathrm{N}-\mathrm{XY}$ and DO Routing on FPGA Platform," IJRECE, pp. 2166-2171, Vol. 6 Issue 3, July-September, 2018. 